\title{
Mean Square Exponential Stability of Stochastic Switched System with Interval Time-Varying Delays
}

\author{
Manlika Rajchakit and Grienggrai Rajchakit
}

Faculty of Science, Maejo University, Chiangmai 50290, Thailand

Correspondence should be addressed to Grienggrai Rajchakit, mrajchakit@yahoo.com

Received 19 March 2012; Revised 5 May 2012; Accepted 9 May 2012

Academic Editor: Miroslava Růžičková

Copyright (c) 2012 M. Rajchakit and G. Rajchakit. This is an open access article distributed under the Creative Commons Attribution License, which permits unrestricted use, distribution, and reproduction in any medium, provided the original work is properly cited.

This paper is concerned with mean square exponential stability of switched stochastic system with interval time-varying delays. The time delay is any continuous function belonging to a given interval, but not necessary to be differentiable. By constructing a suitable augmented LyapunovKrasovskii functional combined with Leibniz-Newton's formula, a switching rule for the mean square exponential stability of switched stochastic system with interval time-varying delays and new delay-dependent sufficient conditions for the mean square exponential stability of the switched stochastic system are first established in terms of LMIs. Numerical example is given to show the effectiveness of the obtained result.

\section{Introduction}

Stability analysis of linear systems with time-varying delays $\dot{x}(t)=A x(t)+D x(t-h(t))$ is fundamental to many practical problems and has received considarable attention [1-11]. Most of the known results on this problem are derived assuming only that the time-varing delay $h(t)$ is a continuously differentiable function, satisfying some boundedness condition on its derivative: $\dot{h}(t) \leq \delta<1$. In delay-dependent stability criteria, the main concern is to enlarge the feasible region of stability criteria in given time-delay interval. Interval timevarying delay means that a time delay varies in an interval in which the lower bound is not restricted to be zero. By constructing a suitable augmented Lyapunov functionals and utilizing free weight matrices, some less conservative conditions for asymptotic stability are derived in [12-21] for systems with time delay varying in an interval. However, the shortcoming of the method used in these works is that the delay function is assumed to be differential and its derivative is still bounded: $\dot{h}(t) \leq \delta$. This paper gives the improved results for the mean square exponential stability of switched stochastic system with interval 
time-varying delay. The time delay is assumed to be a time-varying continuous function belonging to a given interval, but not necessary to be differentiable. Specifically, our goal is to develop a constructive way to design switching rule to the mean square exponential stability of switched stochastic system with interval time-varying delay. By constructing argumented Lyapunov functional combined with LMI technique, we propose new criteria for the mean square exponential stability of the switched stochastic system. The delay-dependent stability conditions are formulated in terms of LMIs.

The paper is organized as follows: Section 2 presents definitions and some wellknown technical propositions needed for the proof of the main results. Delay-dependent mean square exponential stability conditions of the switched stochastic system and numerical example showing the effectiveness of proposed method are presented in Section 3.

\section{Preliminaries}

The following notations will be used in this paper. $R^{+}$denotes the set of all real nonnegative numbers; $R^{n}$ denotes the $n$-dimensional space with the scalar product $\langle\cdot, \cdot\rangle$ and the vector norm $\|\cdot\| ; M^{n \times r}$ denotes the space of all matrices of $(n \times r)$-dimensions; $A^{T}$ denotes the transpose of matrix $A ; A$ is symmetric if $A=A^{T}$; $I$ denotes the identity matrix; $\lambda(A)$ denotes the set of all eigenvalues of $A ; \lambda_{\min / \max }(A)=\min / \max \{\operatorname{Re} \lambda ; \lambda \in \lambda(A)\} ; x_{t}:=\{x(t+s): s \in$ $[-h, 0]\},\left\|x_{t}\right\|=\sup _{s \in[-h, 0]}\|x(t+s)\| ; C\left([0, t], R^{n}\right)$ denotes the set of all $R^{n}$-valued continuous functions on $[0, t]$; matrix $A$ is called semipositive definite $(A \geq 0)$ if $\langle A x, x\rangle \geq 0$, for all $x \in R^{n} ; A$ is positive definite $(A>0)$ if $\langle A x, x\rangle>0$ for all $x \neq 0 ; A>B$ means $A-B>0 . *$ denotes the symmetric term in a matrix.

Consider a switched stochastic system with interval time-varying delay of the form

$$
\begin{gathered}
\dot{x}(t)=A_{\gamma} x(t)+D_{\gamma} x(t-h(t))+\sigma_{\gamma}(x(t), x(k-h(t)), t) \omega(t), \quad t \in R^{+}, \\
x(t)=\phi(t), \quad t \in\left[-h_{2}, 0\right]
\end{gathered}
$$

where $x(t) \in R^{n}$ is the state; $\gamma(\cdot): R^{n} \rightarrow \mathcal{N}:=\{1,2, \ldots, N\}$ is the switching rule, which is a function depending on the state at each time and will be designed. A switching function is a rule which determines a switching sequence for a given switching system. Moreover, $\gamma(x(t))=i$ implies that the system realization is chosen as the $i$ th system, $i=1,2, \ldots, N$. It is seen that the system (2.1) can be viewed as an autonomous switched system in which the effective subsystem changes when the state $x(t)$ hits predefined boundaries. $A_{i}, D_{i} \in$ $M^{n \times n}, i=1,2, \ldots, N$ are given constant matrices, and $\phi(t) \in C\left(\left[-h_{2}, 0\right], R^{n}\right)$ is the initial function with the norm $\|\phi\|=\sup _{s \in\left[-h_{2}, 0\right]}\|\phi(s)\|$.

$\omega(k)$ is a scalar Wiener process (Brownian Motion) on $(\Omega, \mathcal{F}, p)$ with

$$
E\{\omega(t)\}=0, \quad E\left\{\omega^{2}(t)\right\}=1, \quad E\{\omega(i) \omega(j)\}=0 \quad(i \neq j),
$$

and $\sigma_{i}: R^{n} \times R^{n} \times R \rightarrow R^{n}, i=1,2, \ldots, N$ is the continuous function and is assumed to satisfy that

$$
\begin{array}{r}
\sigma_{i}^{T}(x(t), x(t-h(t)), t) \sigma_{i}(x(t), x(t-h(t)), t) \leq \rho_{i 1} x^{T}(t) x(t)+\rho_{i 2} x^{T}(t-h(t)) x(t-h(t)), \\
x(t), x(t-h(t)) \in R^{n},
\end{array}
$$


where $\rho_{i 1}>0$ and $\rho_{i 2}>0, i=1,2, \ldots, N$ are known constant scalars. For simplicity, we denote $\sigma_{i}(x(t), x(t-h(t)), t)$ by $\sigma_{i}$, respectively.

The time-varying delay function $h(t)$ satisfies

$$
0 \leq h_{1} \leq h(t) \leq h_{2}, \quad t \in R^{+} .
$$

The stability problem for switched stochastic system (2.1) is to construct a switching rule that makes the system exponentially stable.

Definition 2.1. Given $\alpha>0$, the switched stochastic system (2.1) is $\alpha$-exponentially stable in the mean square if there exists a switching rule $\gamma(\cdot)$ such that every solution $x(t, \phi)$ of the system satisfies the following condition:

$$
\exists N>0: E\{\|x(t, \phi)\|\} \leq E\left\{N e^{-\alpha t}\|\phi\|\right\}, \quad \forall t \in R^{+} .
$$

We end this section with the following technical well-known propositions, which will be used in the proof of the main results.

Proposition 2.2 (Cauchy inequality). For any symmetric positive definite marix $N \in M^{n \times n}$ and $a, b \in R^{n}$ one has

$$
\pm a^{T} b \leq a^{T} N a+b^{T} N^{-1} b .
$$

Proposition 2.3 (see [22]). For any symmetric positive definite matrix $M \in M^{n \times n}$, scalar $\gamma>0$ and vector function $\omega:[0, \gamma] \rightarrow R^{n}$ such that the integrations concerned are well defined, the following inequality holds:

$$
\left(\int_{0}^{\gamma} \omega(s) d s\right)^{T} M\left(\int_{0}^{\gamma} \omega(s) d s\right) \leq r\left(\int_{0}^{\gamma} \omega^{T}(s) M \omega(s) d s\right) .
$$

Proposition 2.4 (see [23]). Let $E, H$, and $F$ be any constant matrices of appropriate dimensions and $F^{T} F \leq I$. For any $\epsilon>0$, one has

$$
E F H+H^{T} F^{T} E^{T} \leq \epsilon E E^{T}+\epsilon^{-1} H^{T} H .
$$

Proposition 2.5 (Schur complement lemma [24]). Given constant matrices $X, Y, Z$ with appropriate dimensions satisfying $X=X^{T}, Y=Y^{T}>0$. Then $X+Z^{T} Y^{-1} Z<0$ if and only if

$$
\left(\begin{array}{cc}
X & Z^{T} \\
Z & -Y
\end{array}\right)<0 \text { or } \quad\left(\begin{array}{cc}
-Y & Z \\
Z^{T} & X
\end{array}\right)<0
$$




\section{Main Results}

Let us set

$$
\begin{aligned}
& M_{i}=\left(\begin{array}{ccccc}
M_{11} & M_{12} & M_{13} & M_{14} & M_{15} \\
* & M_{22} & 0 & M_{24} & S_{2} \\
* & * & M_{33} & M_{34} & S_{3} \\
* & * & * & M_{44} & M_{45} \\
* & * & * & M_{55}
\end{array}\right), \\
& \lambda_{1}=\lambda_{\min }(P), \\
& \lambda_{2}=\lambda_{\max }(P)+2 h_{2}^{2} \lambda_{\max }(R), \\
& M_{11}=A_{i}^{T} P+P A_{i}-S_{1} A_{i}-A_{i}^{T} S_{1}^{T}+2 \alpha P \\
& \quad-e^{-2 \alpha h_{1}} R-e^{-2 \alpha h_{2}} R+2 \rho_{i 1}, \\
& M_{12}=e^{-2 \alpha h_{1}} R-S_{2} A_{i}, \\
& M_{13}=e^{-2 \alpha h_{2}} R-S_{3} A_{i}, \\
& M_{14}= P D_{i}-S_{1} D_{i}-S_{4} A_{i}, \\
& M_{15}= S_{1}-S_{5} A_{i}, \\
& M_{22}=-e^{-2 \alpha h_{1}} R, \\
& M_{24}= S_{2} D_{i}, \\
& M_{33}=-e^{-2 \alpha h_{2}} R, \\
& M_{34}=-S_{3} D_{i}, \\
& M_{44}=-S_{4} D_{i}+2 \rho_{i 2}, \\
& M_{45}= S_{4}-S_{5} D_{i}, \\
& M_{55}= S_{5}+S_{5}^{T}+h_{1}^{2} R+h_{2}^{2} R . \\
& M_{1}
\end{aligned}
$$

The main result of this paper is summarized in the following theorem.

Theorem 3.1. Given $\alpha>0$, the zero solution of the switched stochastic system (2.1) is $\alpha$ exponentially stable in the mean square if there exist symmetric positive definite matrices $P, R$, and matrices $S_{i}, i=1,2, \ldots, 5$ satisfying the following conditions:

(i) $\mathcal{M}_{i}<0, i=1,2, \ldots, N$.

The switching rule is chosen as $\gamma(x(t))=i$. Moreover, the solution $x(t, \phi)$ of the switched stochastic system satisfies

$$
E\{\|x(t, \phi)\|\} \leq E\left\{\sqrt{\frac{\lambda_{2}}{\lambda_{1}}} e^{-\alpha t}\|\phi\|\right\}, \quad \forall t \in R^{+} .
$$


Proof. We consider the following Lyapunov-Krasovskii functional for the system (2.1):

$$
E\left\{V\left(t, x_{t}\right)\right\}=\sum_{i=1}^{3} E\left\{V_{i}\right\}
$$

where

$$
\begin{aligned}
& V_{1}=x^{T}(t) P x(t), \\
& V_{2}=h_{1} \int_{-h_{1}}^{0} \int_{t+s}^{t} e^{2 \alpha(\tau-t)} \dot{x}^{T}(\tau) R \dot{x}(\tau) d \tau d s, \\
& V_{3}=h_{2} \int_{-h_{2}}^{0} \int_{t+s}^{t} e^{2 \alpha(\tau-t)} \dot{x}^{T}(\tau) R \dot{x}(\tau) d \tau d s .
\end{aligned}
$$

It easy to check that

$$
E\left\{\lambda_{1}\|x(t)\|^{2}\right\} \leq E\left\{V\left(t, x_{t}\right)\right\} \leq E\left\{\lambda_{2}\left\|x_{t}\right\|^{2}\right\}, \quad \forall t \geq 0,
$$

Taking the derivative of Lyapunov-Krasovskii functional along the solution of system (2.1) and taking the mathematical expectation, we obtained

$$
\begin{aligned}
E\left\{\dot{V}_{1}\right\} & =E\left\{2 x^{T}(t) P \dot{x}(t)\right\} \\
& =E\left\{x^{T}(t)\left[A_{i}^{T} P+A_{i} P\right] x(t)+2 x^{T}(t) P D_{i} x(t-h(t))+2 x^{T}(t) P \sigma_{i} \omega(t)\right\}, \\
E\left\{\dot{V}_{2}\right\} & =E\left\{h_{1}^{2} \dot{x}^{T}(t) R \dot{x}(t)-h_{1} e^{-2 \alpha h_{1}} \int_{t-h_{1}}^{t} \dot{x}^{T}(s) R \dot{x}(s) d s-2 \alpha V_{2}\right\}, \\
E\left\{\dot{V}_{3}\right\} & =E\left\{h_{2}^{2} \dot{x}^{T}(t) R \dot{x}(t)-h_{2} e^{-2 \alpha h_{2}} \int_{t-h_{2}}^{t} \dot{x}^{T}(s) R \dot{x}(s) d s-2 \alpha V_{3}\right\} .
\end{aligned}
$$

Applying Proposition 2.3 and the Leibniz-Newton formula, we have

$$
\begin{aligned}
E\left\{-h_{i} \int_{t-h_{i}}^{t} \dot{x}^{T}(s) R \dot{x}(s) d s\right\} & \leq E\left\{-\left[\int_{t-h_{i}}^{t} \dot{x}(s) d s\right]^{T} R\left[\int_{t-h_{i}}^{t} \dot{x}(s) d s\right]\right\} \\
& \leq E\left\{-\left[x(t)-x\left(t-h_{i}\right)\right]^{T} R\left[x(t)-x\left(t-h_{i}\right)\right]\right\} \\
& =E\left\{-x^{T}(t) R x(t)+2 x^{T}(t) R x\left(t-h_{i}\right)-x^{T}\left(t-h_{i}\right) R x\left(t-h_{i}\right)\right\},
\end{aligned}
$$


Therefore, we have

$$
\begin{aligned}
E\{\dot{V}(\cdot)+2 \alpha V(\cdot)\} \leq & E\left\{x^{T}(t)\left[A_{i}^{T} P+A_{i} P+2 \alpha P\right] x(t)\right\} \\
& +E\left\{2 x^{T}(t) P D_{i} x(t-h(t))+2 x^{T}(t) P \sigma_{i} \omega(t)\right\} \\
& +E\left\{\dot{x}^{T}(t)\left[\left(h_{1}^{2}+h_{2}^{2}\right) R\right] \dot{x}(t)\right\} \\
& -E\left\{e^{-2 \alpha h_{1}}\left[x(t)-x\left(t-h_{1}\right)\right]^{T} R\left[x(t)-x\left(t-h_{1}\right)\right]\right\} \\
& -E\left\{e^{-2 \alpha h_{2}}\left[x(t)-x\left(t-h_{2}\right)\right]^{T} R\left[x(t)-x\left(t-h_{2}\right)\right]\right\} .
\end{aligned}
$$

By using the following identity relation

$$
\dot{x}(t)-A_{i} x(t)-D_{i} x(t-h(t))=0,
$$

we have

$$
\begin{aligned}
& 2 x^{T}(t) S_{1} \dot{x}(t)-2 x^{T}(t) S_{1} A_{i} x(t)-2 x^{T}(t) S_{1} D_{i} x(t-h(t))-2 x^{T}(t) S_{1} \sigma_{i} \omega(t)=0, \\
& 2 x^{T}\left(t-h_{1}\right) S_{2} \dot{x}(t)-2 x^{T}\left(t-h_{1}\right) S_{2} A_{i} x(t)-2 x^{T}\left(t-h_{1}\right) S_{2} D_{i} x(t-h(t)) \\
& \quad-2 x^{T}\left(t-h_{1}\right) S_{2} \sigma_{i} \omega(t)=0, \\
& 2 x^{T}\left(t-h_{2}\right) S_{3} \dot{x}(t)-2 x^{T}\left(t-h_{2}\right) S_{3} A_{i} x(t)-2 x^{T}\left(t-h_{2}\right) S_{3} D_{i} x(t-h(t)) \\
& \quad-2 x^{T}\left(t-h_{2}\right) S_{3} \sigma_{i} \omega(t)=0, \\
& 2 x^{T}(t-h(t)) S_{4} \dot{x}(t)-2 x^{T}(t-h(t)) S_{4} A_{i} x(t)-2 x^{T}(t-h(t)) S_{4} D_{i} x(t-h(t)) \\
& \quad-2 x^{T}(t-h(t)) S_{4} \sigma_{i} \omega(t)=0, \\
& 2 \dot{x}^{T}(t) S_{5} \dot{x}(t)-2 \dot{x}^{T}(t) S_{5} A_{i} x(t)-2 \dot{x}^{T}(t) S_{5} D_{i} x(t-h(t))-2 \dot{x}^{T}(t) S_{5} \sigma_{i} \omega(t)=0, \\
& 2 \omega^{T}(t) \sigma_{i}^{T} \dot{x}(t)-2 \omega^{T}(t) \sigma_{i}^{T} A_{i} x(t)-2 \omega^{T}(t) \sigma_{i}^{T} D_{i} x(t-h(t))-2 \omega^{T}(t) \sigma_{i}^{T} \sigma_{i} \omega(t)=0 .
\end{aligned}
$$

Adding all the zero items of (3.10) into (3.8), we obtain

$$
\begin{aligned}
E\{\dot{V}(\cdot)+2 \alpha V(\cdot)\} \leq & E\left\{x^{T}(t)\left[A_{i}^{T} P+P A_{i}+2 \alpha P-e^{-2 \alpha h_{1}} R\right] x(t)\right\} \\
& -E\left\{x^{T}(t)\left[e^{-2 \alpha h_{2}} R-S_{1} A_{i}-A_{i}^{T} S_{1}^{T}\right] x(t)\right\} \\
& +E\left\{2 x^{T}(t)\left[e^{-2 \alpha h_{1}} R-S_{2} A_{i}\right] x\left(t-h_{1}\right)\right\} \\
& +E\left\{2 x^{T}(t)\left[e^{-2 \alpha h_{2}} R-S_{3} A_{i}\right] x\left(t-h_{2}\right)\right\} \\
& +E\left\{2 x^{T}(t)\left[P D_{i}-S_{1} D_{i}-S_{4} A_{i}\right] x(t-h(t))\right\}
\end{aligned}
$$


Abstract and Applied Analysis

$$
\begin{aligned}
& +E\left\{2 x^{T}(t)\left[S_{1}-S_{5} A_{i}\right] \dot{x}(t)\right\} \\
& +E\left\{2 x^{T}(t)\left[P \sigma_{i}-S_{1} \sigma_{i}-A_{i}^{T} \sigma_{i}\right] \omega(t)\right\} \\
& +E\left\{x^{T}\left(t-h_{1}\right)\left[-e^{-2 \alpha h_{1}} R\right] x\left(t-h_{1}\right)\right\} \\
& +E\left\{2 x^{T}\left(t-h_{1}\right)\left[-S_{2} D_{i}\right] x(t-h(t))\right\} \\
& +E\left\{2 x^{T}\left(t-h_{1}\right) S_{2} \dot{x}(t)+2 x^{T}\left(t-h_{1}\right)\left[-S_{2} \sigma_{i}\right] \omega(t)\right\} \\
& +E\left\{x^{T}\left(t-h_{2}\right)\left[-e^{-2 \alpha h_{2}} R\right] x\left(t-h_{2}\right)\right\} \\
& +E\left\{x^{T}\left(t-h_{2}\right)\left[-S_{3} D_{i}\right] x(t-h(t))\right\} \\
& +E\left\{2 x^{T}\left(t-h_{2}\right) S_{3} \dot{x}(t)+2 x^{T}\left(t-h_{2}\right)\left[-S_{3} \sigma_{i}\right] \omega(t)\right\} \\
& +E\left\{x^{T}(t-h(t))\left[-S_{4} D_{i}\right] x(t-h(t))\right\} \\
& +E\left\{2 x^{T}(t-h(t))\left[S_{4}-S_{5} D_{i}\right] \dot{x}(t)\right\} \\
& +E\left\{2 x^{T}(t-h(t))\left[-S_{4} \sigma_{i}-D_{i}^{T} \sigma_{i}\right] \omega(t)\right\} \\
& +E\left\{\dot{x}^{T}(t)\left[S_{5}+S_{5}^{T}+h_{1}^{2} R+h_{2}^{2} R\right] \dot{x}(t)\right\} \\
& +E\left\{2 \dot{x}^{T}(t)\left[\sigma_{i}^{T}-S_{5} \sigma_{i}\right] \omega(t)\right\} \\
& +E\left\{2 \omega^{T}(t)\left[-\sigma_{i} \sigma_{i}\right] \omega(t)\right\} .
\end{aligned}
$$

By assumption (2.2), we have

$$
\begin{aligned}
E\{\dot{V}(\cdot)+2 \alpha V(\cdot)\} \leq & E\left\{x^{T}(t)\left[A_{i}^{T} P+P A_{i}+2 \alpha P-e^{-2 \alpha h_{1}} R\right] x(t)\right\} \\
& -E\left\{x^{T}(t)\left[e^{-2 \alpha h_{2}} R-S_{1} A_{i}-A_{i}^{T} S_{1}^{T}\right] x(t)\right\} \\
& +E\left\{2 x^{T}(t)\left[e^{-2 \alpha h_{1}} R-S_{2} A_{i}\right] x\left(t-h_{1}\right)\right\} \\
& +E\left\{2 x^{T}(t)\left[e^{-2 \alpha h_{2}} R-S_{3} A_{i}\right] x\left(t-h_{2}\right)\right\} \\
& +E\left\{2 x^{T}(t)\left[P D_{i}-S_{1} D_{i}-S_{4} A_{i}\right] x(t-h(t))\right\} \\
& +E\left\{2 x^{T}(t)\left[S_{1}-S_{5} A_{i}\right] \dot{x}(t)\right\} \\
& +E\left\{x^{T}\left(t-h_{1}\right)\left[-e^{-2 \alpha h_{1}} R\right] x\left(t-h_{1}\right)\right\}
\end{aligned}
$$




$$
\begin{aligned}
& +E\left\{2 x^{T}\left(t-h_{1}\right)\left[-S_{2} D_{i}\right] x(t-h(t))\right\} \\
& +E\left\{x^{T}\left(t-h_{2}\right)\left[-e^{-2 \alpha h_{2}} R\right] x\left(t-h_{2}\right)\right\} \\
& +E\left\{x^{T}\left(t-h_{2}\right)\left[-S_{3} D_{i}\right] x(t-h(t))\right\} \\
& +E\left\{2 x^{T}\left(t-h_{2}\right) S_{3} \dot{x}(t)\right\} \\
& +E\left\{x^{T}(t-h(t))\left[-S_{4} D_{i}\right] x(t-h(t))\right\} \\
& +E\left\{2 x^{T}(t-h(t))\left[S_{4}-S_{5} D_{i}\right] \dot{x}(t)\right\} \\
& +E\left\{\dot{x}^{T}(t)\left[S_{5}+S_{5}^{T}+h_{1}^{2} R+h_{2}^{2} R\right] \dot{x}(t)\right\} \\
& +E\left\{2\left[-\sigma_{i}^{T} \sigma_{i}\right]\right\} .
\end{aligned}
$$

Applying assumption (2.3), the following estimations hold:

$$
\begin{aligned}
E\{\dot{V}(\cdot)+2 \alpha V(\cdot)\} \leq & E\left\{x^{T}(t)\left[A_{i}^{T} P+P A_{i}+2 \alpha P-e^{-2 \alpha h_{1}} R\right] x(t)\right\} \\
& -E\left\{x^{T}(t)\left[e^{-2 \alpha h_{2}} R-S_{1} A_{i}-A_{i}^{T} S_{1}^{T}+2 \rho_{i 1} I\right] x(t)\right\} \\
& +E\left\{2 x^{T}(t)\left[e^{-2 \alpha h_{1}} R-S_{2} A_{i}\right] x\left(t-h_{1}\right)\right\} \\
& +E\left\{2 x^{T}(t)\left[e^{-2 \alpha h_{2}} R-S_{3} A_{i}\right] x\left(t-h_{2}\right)\right\} \\
& +E\left\{2 x^{T}(t)\left[P D_{i}-S_{1} D_{i}-S_{4} A_{i}\right] x(t-h(t))\right\} \\
& +E\left\{2 x^{T}(t)\left[S_{1}-S_{5} A_{i}\right] \dot{x}(t)\right\} \\
& +E\left\{x^{T}\left(t-h_{1}\right)\left[-e^{-2 \alpha h_{1}} R\right] x\left(t-h_{1}\right)\right\} \\
& +E\left\{2 x^{T}\left(t-h_{1}\right)\left[-S_{2} D_{i}\right] x(t-h(t))\right\} \\
& +E\left\{x^{T}\left(t-h_{2}\right)\left[-e^{-2 \alpha h_{2}} R\right] x\left(t-h_{2}\right)\right\} \\
& +E\left\{x^{T}\left(t-h_{2}\right)\left[-S_{3} D_{i}\right] x(t-h(t))\right\} \\
& +E\left\{2 x^{T}\left(t-h_{2}\right) S_{3} \dot{x}(t)\right\} \\
& +E\left\{x^{T}(t-h(t))\left[-S_{4} D_{i}+2 \rho_{i 2} I\right] x(t-h(t))\right\} \\
& +E\left\{2 x^{T}(t-h(t))\left[S_{4}-S_{5} D_{i}\right] \dot{x}(t)\right\}
\end{aligned}
$$


Abstract and Applied Analysis

$$
\begin{aligned}
& +E\left\{\dot{x}^{T}(t)\left[S_{5}+S_{5}^{T}+h_{1}^{2} R+h_{2}^{2} R\right] \dot{x}(t)\right\} \\
= & E\left\{\zeta^{T}(t) M_{i} \zeta(t)\right\},
\end{aligned}
$$

where

$$
\begin{aligned}
\zeta(t)= & {\left[x(t), x\left(t-h_{1}\right), x\left(t-h_{2}\right), x(t-h(t)), \dot{x}(t)\right], } \\
M_{i}= & {\left[\begin{array}{ccccc}
M_{11} & M_{12} & M_{13} & M_{14} & M_{15} \\
* & M_{22} & 0 & M_{24} & S_{2} \\
* & * & M_{33} & M_{34} & S_{3} \\
* & * & * & M_{44} & M_{45} \\
* & * & * & M_{55}
\end{array}\right], } \\
M_{11}= & A_{i}^{T} P+P A_{i}-S_{1} A_{i}-A_{i}^{T} S_{1}^{T} \\
& +2 \alpha P-e^{-2 \alpha h_{1}} R-e^{-2 \alpha h_{2}} R+2 \rho_{i 1}, \\
M_{12}= & e^{-2 \alpha h_{1}} R-S_{2} A_{i}, \\
M_{13}= & e^{-2 \alpha h_{2}} R-S_{3} A_{i \prime} \\
M_{14}= & P D_{i}-S_{1} D_{i}-S_{4} A_{i}, \\
M_{15}= & S_{1}-S_{5} A_{i}, \\
M_{22}= & -e^{-2 \alpha h_{1}} R \\
M_{24}= & -S_{2} D_{i}, \\
M_{33}= & -e^{-2 \alpha h_{2}} R \\
M_{34}= & -S_{3} D_{i}, \\
M_{44}= & -S_{4} D_{i}+2 \rho_{i 2} I \\
M_{45}= & S_{4}-S_{5} D_{i}, \\
M_{55}= & S_{5}+S_{5}^{T}+h_{1}^{2} R+h_{2}^{2} R .
\end{aligned}
$$

Therefore, we finally obtain from (3.13) and the condition (i) that

$$
E\{\dot{V}(\cdot)+2 \alpha V(\cdot)\}<0, \quad \forall i=1,2, \ldots, N, t \in R^{+},
$$

and hence

$$
E\left\{\dot{V}\left(t, x_{t}\right)\right\} \leq-E\left\{2 \alpha V\left(t, x_{t}\right)\right\}, \quad \forall t \in R^{+} .
$$


Integrating both sides of (3.16) from 0 to $t$, we obtain

$$
E\left\{V\left(t, x_{t}\right)\right\} \leq E\left\{V(\phi) e^{-2 \alpha t}\right\}, \quad \forall t \in R^{+}
$$

Furthermore, taking condition (3.5) into account, we have

$$
E\left\{\lambda_{1}\|x(t, \phi)\|^{2}\right\} \leq E\left\{V\left(x_{t}\right)\right\} \leq E\left\{V(\phi) e^{-2 \alpha t}\right\} \leq E\left\{\lambda_{2} e^{-2 \alpha t}\|\phi\|^{2}\right\}
$$

then

$$
E\{\|x(t, \phi)\|\} \leq E\left\{\sqrt{\frac{\lambda_{2}}{\lambda_{1}}} e^{-\alpha t}\|\phi\|\right\}, \quad t \in R^{+},
$$

By Definition 2.1, the system (2.1) is exponentially stable in the mean square. The proof is complete.

To illustrate the obtained result, let us give the following numerical examples.

Example 3.2. Consider the following the switched stochastic systems with interval timevarying delay (2.1), where the delay function $h(t)$ is given by

$$
\begin{gathered}
h(t)=0.1+0.8311 \sin ^{2} 3 t \\
A_{1}=\left(\begin{array}{cc}
-1 & 0.01 \\
0.02 & -2
\end{array}\right), \quad A_{2}=\left(\begin{array}{cc}
-1.1 & 0.02 \\
0.01 & -2
\end{array}\right), \\
D_{1}=\left(\begin{array}{cc}
-0.1 & 0.01 \\
0.02 & -0.3
\end{array}\right), \quad D_{2}=\left(\begin{array}{cc}
-0.1 & 0.02 \\
0.01 & -0.2
\end{array}\right) .
\end{gathered}
$$

It is worth noting that the delay function $h(t)$ is nondifferentiable. Therefore, the methods used is in [2-15] are not applicable to this system. By LMI toolbox of MATLAB, by using LMI Toolbox in MATLAB, the LMI (i) is feasible with $h_{1}=0.1, h_{2}=0.9311, \alpha=0.1, \rho_{11}=$ $0.01, \rho_{12}=0.01, \rho_{21}=0.01, \rho_{22}=0.01$, and

$$
\begin{gathered}
P=\left(\begin{array}{cc}
2.0788 & -0.0135 \\
-0.0135 & 1.5086
\end{array}\right), \quad R=\left(\begin{array}{cc}
1.0801 & -0.0042 \\
-0.0042 & 0.8450
\end{array}\right), \\
S_{1}=\left(\begin{array}{cc}
-0.6210 & -0.0335 \\
0.0499 & -0.3576
\end{array}\right), \quad S_{2}=\left(\begin{array}{cc}
-0.3602 & 0.0170 \\
0.0298 & -0.3550
\end{array}\right), \\
S_{3}=\left(\begin{array}{cc}
-0.3602 & 0.0170 \\
0.0298 & -0.3550
\end{array}\right), \quad S_{4}=\left(\begin{array}{cc}
0.6968 & -0.0401 \\
-0.0525 & 0.7040
\end{array}\right), \quad S_{5}=\left(\begin{array}{cc}
-1.4043 & 0.0265 \\
-0.0028 & -0.9774
\end{array}\right)
\end{gathered}
$$


By Theorem 3.1 the switched stochastic systems (2.1) are 0.1-exponentially stable in the mean square and the switching rule is chosen as $\gamma(x(t))=i$. Moreover, the solution $x(t, \phi)$ of the system satisfies

$$
E\{\|x(t, \phi)\|\} \leq E\left\{1.8731 e^{-0.1 t}\|\phi\|\right\}, \quad \forall t \in R^{+} .
$$

\section{Conclusions}

In this paper, we have proposed new delay-dependent conditions for the mean square exponential stability of switched stochastic system with non-differentiable interval timevarying delay. By constructing a set of improved Lyapunov-Krasovskii functionals and Newton-Leibniz formula, the conditions for the exponential stability of the systems have been established in terms of LMIs.

\section{Acknowledgments}

This work was supported by the Thai Research Fund Grant, the Higher Education Commission and Faculty of Science, Maejo University, Thailand.

\section{References}

[1] M. C. de Oliveira, J. C. Geromel, and L. Hsu, "LMI characterization of structural and robust stability: the discrete-time case," Linear Algebra and Its Applications, vol. 296, no. 1-3, pp. 27-38, 1999.

[2] Y.-J. Sun, "Global stabilizability of uncertain systems with time-varying delays via dynamic observerbased output feedback," Linear Algebra and Its Applications, vol. 353, pp. 91-105, 2002.

[3] I. A. Dzhalladova, J. Baštinec, J. Diblík, and D. Y. Khusainov, "Estimates of exponential stability for solutions of stochastic control systems with delay," Abstract and Applied Analysis, vol. 2011, Article ID 920412, 14 pages, 2011.

[4] D. Y. Khusainov, J. Diblík, Z. Svoboda, and Z. Šmarda, "Instable trivial solution of autonomous differential systems with quadratic right-hand sides in a cone," Abstract and Applied Analysis, vol. 2011, Article ID 154916, 23 pages, 2011.

[5] J. Diblík, D. Y. Khusainov, I. V. Grytsay, and Z. Šmarda, "Stability of nonlinear autonomous quadratic discrete systems in the critical case," Discrete Dynamics in Nature and Society, vol. 2010, Article ID 539087, 23 pages, 2010.

[6] J. Diblík, D. Y. Khusainov, and M. Růžičková, "Solutions of Discrete equations with prescrid asymptotic behavior," Dynamic Systems and Applications, vol. 4, pp. 395-402, 2004.

[7] J. Diblík, D. Y. Khusainov, and I. V. Grytsay, "Stability investigation of nonlinear quadratic discrete dynamics systems in the critical case," Journal of Physics, vol. 96, no. 1, Article ID 012042, 2008.

[8] J. Baštinec, J. Diblík, D. Y. Khusainov, and A. Ryvolová, "Exponential stability and estimation of solutions of linear differential systems of neutral type with constant coefficients," Boundary Value Problems, vol. 2010, Article ID 956121, 20 pages, 2010.

[9] J. Diblík and I. Hlavičková, "Combination of Liapunov and retract methods in the investigation of the asymptotic behavior of solutions of systems of discrete equations," Dynamic Systems and Applications, vol. 18, no. 3-4, pp. 507-537, 2009.

[10] J. Baštinec, J. Diblík, and Z. Šmarda, “Existence of positive solutions of discrete linear equations with a single delay," Journal of Difference Equations and Applications, vol. 16, no. 9, pp. 1047-1056, 2010.

[11] J. Diblík, M. Růžičková, and Z. Šmarda, “Ważewski's method for systems of dynamic equations on time scales," Nonlinear Analysis: Theory, Methods \& Applications, vol. 71, no. 12, pp. e1124-e1131, 2009.

[12] O. M. Kwon and J. H. Park, "Delay-range-dependent stabilization of uncertain dynamic systems with interval time-varying delays," Applied Mathematics and Computation, vol. 208, no. 1, pp. 58-68, 2009. 
[13] H. Shao, "New delay-dependent stability criteria for systems with interval delay," Automatica, vol. 45, no. 3, pp. 744-749, 2009.

[14] J. Sun, G. P. Liu, J. Chen, and D. Rees, "Improved delay-range-dependent stability criteria for linear systems with time-varying delays," Automatica, vol. 46, no. 2, pp. 466-470, 2010.

[15] W. Zhang, X.-S. Cai, and Z.-Z. Han, "Robust stability criteria for systems with interval time-varying delay and nonlinear perturbations," Journal of Computational and Applied Mathematics, vol. 234, no. 1, pp. 174-180, 2010.

[16] V. N. Phat, "Robust stability and stabilizability of uncertain linear hybrid systems with state delays," IEEE Transactions on Circuits and Systems II, vol. 52, no. 2, pp. 94-98, 2005.

[17] V. N. Phat and P. T. Nam, "Exponential stability and stabilization of uncertain linear time-varying systems using parameter dependent Lyapunov function," International Journal of Control, vol. 80, no. 8, pp. 1333-1341, 2007.

[18] V. N. Phat and P. Niamsup, "Stability analysis for a class of functional differential equations and applications," Nonlinear Analysis: Theory, Methods \& Applications, vol. 71, no. 12, pp. 6265-6275, 2009.

[19] V. N. Phat, Y. Khongtham, and K. Ratchagit, "LMI approach to exponential stability of linear systems with interval time-varying delays," Linear Algebra and Its Applications, vol. 436, no. 1, pp. 243-251, 2012.

[20] K. Ratchagit and V. N. Phat, "Stability criterion for discrete-time systems," Journal of Inequalities and Applications, vol. 2010, Article ID 201459, 6 pages, 2010.

[21] F. Uhlig, "A recurring theorem about pairs of quadratic forms and extensions: a survey," Linear Algebra and Its Applications, vol. 25, pp. 219-237, 1979.

[22] K. Gu, "An integral inequality in the stability problem of time-delay systems," in Proceedings of the 39th IEEE Confernce on Decision and Control, vol. 3, pp. 2805-2810, IEEE Publisher, New York, NY, USA, 2000.

[23] Y. Wang, L. Xie, and C. E. de Souza, “Robust control of a class of uncertain nonlinear systems," Systems $\mathcal{E}$ Control Letters, vol. 19, no. 2, pp. 139-149, 1992.

[24] S. Boyd, L. El Ghaoui, E. Feron, and V. Balakrishnan, Linear Matrix Inequalities in System and Control Theory, vol. 15 of SIAM Studies in Applied Mathematics, Society for Industrial and Applied Mathematics (SIAM), Philadelphia, Pa, USA, 1994. 


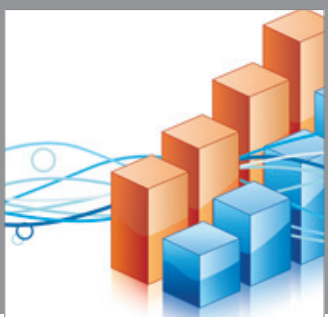

Advances in

Operations Research

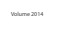

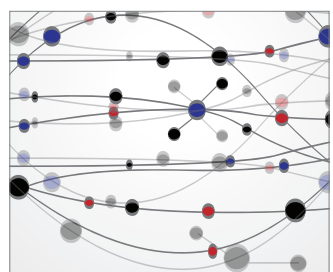

\section{The Scientific} World Journal
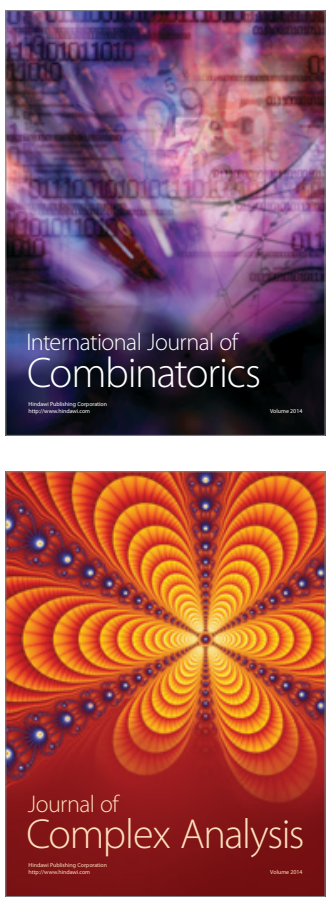

International Journal of

Mathematics and

Mathematical

Sciences
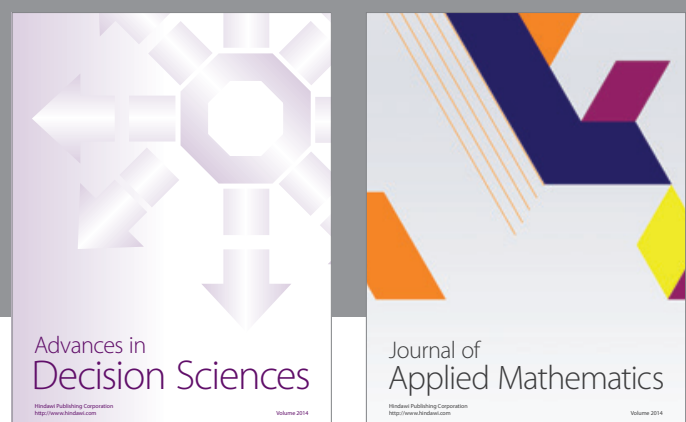

Journal of

Applied Mathematics
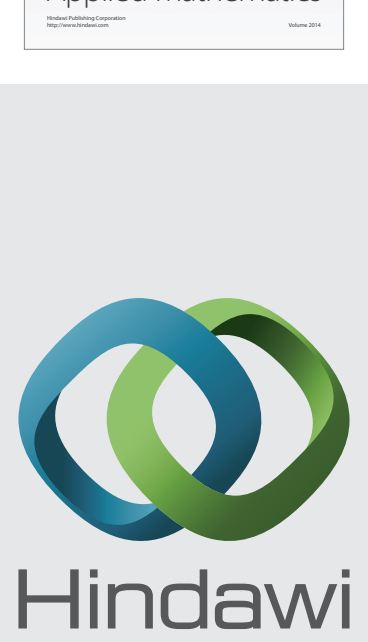

Submit your manuscripts at http://www.hindawi.com
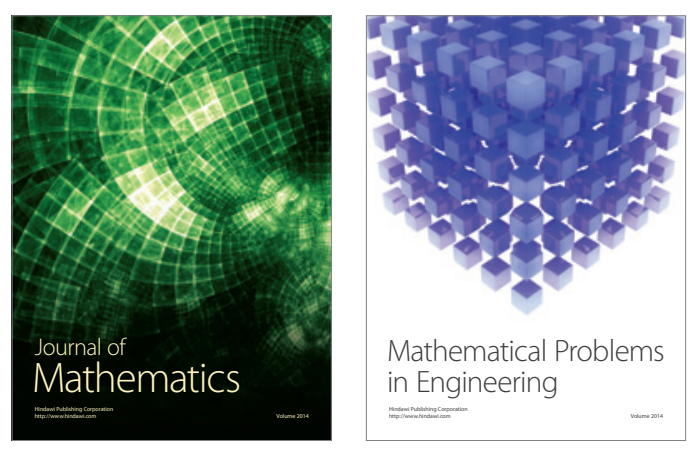

Mathematical Problems in Engineering
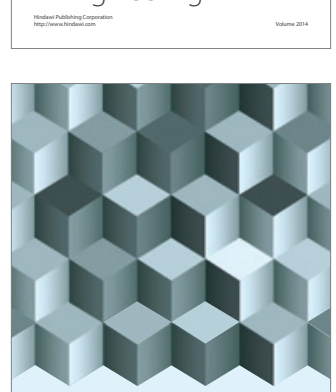

Journal of

Function Spaces
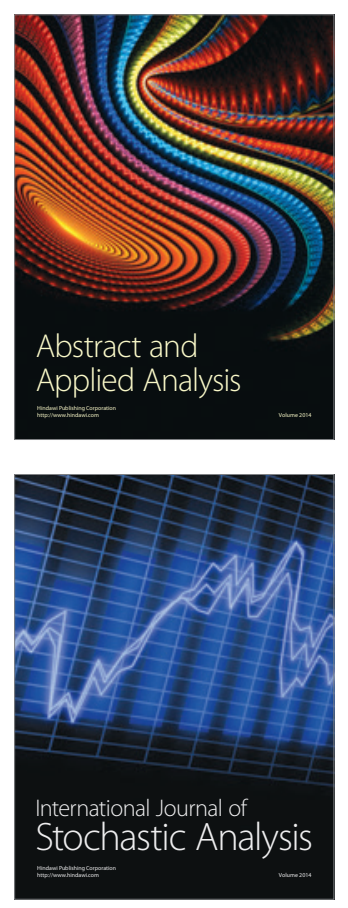

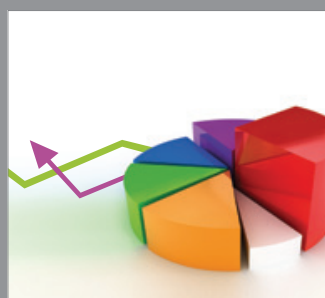

ournal of

Probability and Statistics

Promensencen
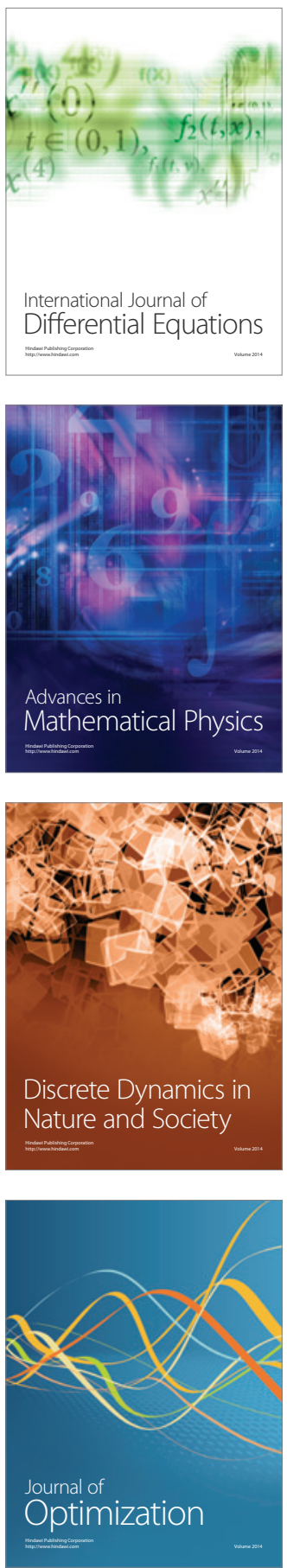